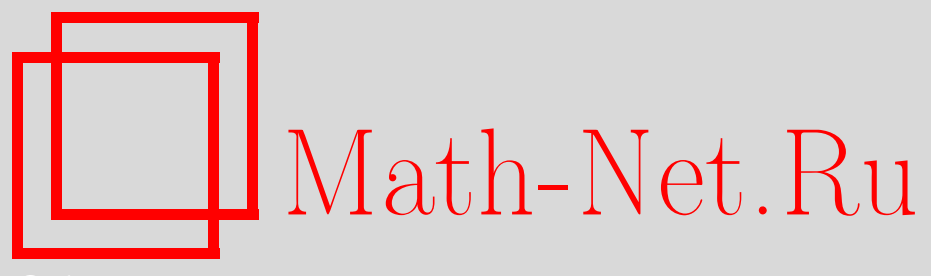

M. Е. Широков, О супераддитивности выпуклого замыкания выходной энтропии квантового канала, УМН, 2006, том 61, выпуск 6, 191-192

DOI: https://doi.org/10.4213/rm5582

Использование Общероссийского математического портала Math-Net.Ru подразумевает, что вы прочитали и согласны с пользовательским соглашением http://www . mathnet.ru/rus/agreement

Параметры загрузки:

IP : 35.173 .219 .149

26 апреля 2023 г., 16:36:33

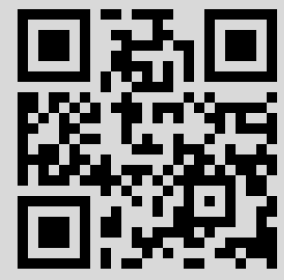




\title{
О супераддитивности выпуклого замыкания выходной энтропии квантового канала
}

\author{
М. Е. Широков
}

Одним из основных достижений квантовой теории информации (см. [1]) последних лет является доказательство эквивалентности различных гипотез (суб-, супер-) аддитивности для конечномерных квантовых каналов и систем [2] (см. обзор в [3]). Основной целью данной статьи является обобщение этого результата на бесконечномерный случай.

Пусть $\mathscr{H}, \mathscr{H}^{\prime}$ - сепарабельные гильбертовы пространства. Квантовым каналом называется линейное вполне положительное сохраняющее след отображение $\Phi: \mathfrak{T}(\mathscr{H}) \mapsto$ $\mathfrak{T}\left(\mathscr{H}^{\prime}\right)$, где $\mathfrak{T}(\mathscr{H})$ - идеал всех ядерных операторов в $\mathscr{H}$. В частности, $\Phi$ порождает аффинное отображение выпуклого множества $\mathfrak{S}(\mathscr{H})$ состояний (операторов плотности) на пространстве $\mathscr{H}$ в множество $\mathfrak{S}\left(\mathscr{H}^{\prime}\right)$ состояний на пространстве $\mathscr{H}^{\prime}$ [1].

Важными характеристиками квантового канала являются его выходная энтропия $H_{\Phi}(\rho)=H(\Phi(\rho))$ - вогнутая полунепрерывная снизу функция на пространстве состояний входной системы с множеством значений $[0,+\infty]-$ и ее выпуклое замыкание (см. [4]), которое будем обозначать $\widehat{H}_{\Phi}(\rho)$ и называть $\widehat{H}$-функцией канала $\Phi$. В [5] показано, что выпуклое замыкание выходной энтропии произвольного квантового канала определяется выражением

$$
\widehat{H}_{\Phi}(\rho)=\inf _{\mu} \int_{\mathfrak{S}(\mathscr{H})} H_{\Phi}(\sigma) \mu(d \sigma),
$$

в котором точная нижняя грань берется по множеству всех вероятностных мер на $\mathfrak{S}(\mathscr{H})$ с барицентром $\rho$, и что эта нижняя грань всегда достигается на некоторой мере с носителем на множестве чистых состояний.

В [5] (предложение 7) получено достаточное условие непрерывности $\widehat{H}$-функции бесконечномерного канала $\Phi$ на подмножестве состояний, которое равносильно следующему утверждению:

$$
\left\{\lim _{n \rightarrow+\infty} H_{\Phi}\left(\rho_{n}\right)=H_{\Phi}\left(\rho_{0}\right)<+\infty\right\} \Rightarrow\left\{\lim _{n \rightarrow+\infty} \widehat{H}_{\Phi}\left(\rho_{n}\right)=\widehat{H}_{\Phi}\left(\rho_{0}\right)\right\} .
$$

Свойство супераддитивности $\widehat{H}$ - $у$ укции для каналов $\Phi$ и $\Psi$ состоит в выполнении неравенства

$$
\widehat{H}_{\Phi \otimes \Psi}(\omega) \geqslant \widehat{H}_{\Phi}\left(\omega^{\mathscr{H}}\right)+\widehat{H}_{\Psi}\left(\omega^{\mathscr{K}}\right)
$$

для всех $\omega \in \mathfrak{S}(\mathscr{H} \otimes \mathscr{K})$, где $\omega^{\mathscr{H}}=\operatorname{Tr}_{\mathscr{K}} \omega$ и $\omega^{\mathscr{K}}=\operatorname{Tr}_{\mathscr{H}} \omega$. Данное свойство гарантирует аддитивность минимальной выходной энтропии для каналов $\Phi$ и $\Psi$ (см. [1], [3]). Если $\Phi$ и $\Psi$ - частичные следы, то супераддитивность $\widehat{H}$-функции для этих каналов означает супераддитивность "сцепленности формирования" EoF (Entanglement of Formation) - важной характеристики состояния составной квантовой системы, являющейся мерой его сцепленности (см. [1], [5]).

Для конечномерных каналов $\Phi$ и $\Psi$ супераддитивность $\widehat{H}$-функции равносильна аддитивности х-пропускной способности этих каналов с произвольными ограничениями [6]. Одним из препятствий для доказательства аналогичного утверждения для бесконечномерных каналов является существование суперсцепленных состояний - чистых состояний составной системы, имеющих частичные следы с бесконечной энтропией (см. замечание 4 в [7]). Именно наличие таких состояний не позволяло до сих пор доказать супераддитивность $\widehat{H}$-функции (и даже аддитивность минимальной выходной энтропии) для некоторых классов бесконечномерных каналов, для которых аддитивность $\chi$-пропускной способности с произвольными ограничениями выведена в [7] из соответствующих конечномерных утверждений [6], [8].

Работа выполнена при поддержке научной программы Отделения математических наук РАН “Современные проблемы теоретической математики” и РФФИ (проект № 06-01-00164-а). 
Частично проблему суперсцепленных состояний удается преодолеть с помощью следующего утверждения, играющего ключевую роль в доказательстве всех приведенных ниже результатов, которое получено на основе свойства непрерывности (2) и некоторых других результатов из [5].

Лемма. Пусть $\Phi: \mathfrak{S}(\mathscr{H}) \mapsto \mathfrak{S}\left(\mathscr{H}^{\prime}\right) u \Psi: \mathfrak{S}(\mathscr{K}) \mapsto \mathfrak{S}\left(\mathscr{K}^{\prime}\right)-$ произвольные квантовые каналы. Неравенство (3) имеет место для всех состояний $\omega$ из $\mathfrak{S}(\mathscr{H} \otimes \mathscr{K})$, если для любого состояния конечного ранга $\omega_{0}$ из $\mathfrak{S}(\mathscr{H} \otimes \mathscr{K})$ с конечной выходной энтропией $H_{\Phi \otimes \Psi}\left(\omega_{0}\right)$ существует последовательность $\left\{\omega_{n}\right\}$ состолний из $\mathfrak{S}(\mathscr{H} \otimes \mathscr{K})$ со свойствами: (i) $\lim _{n \rightarrow+\infty} \omega_{n}=\omega_{0} u \lim _{n \rightarrow+\infty} H_{\Phi \otimes \Psi}\left(\omega_{n}\right)=H_{\Phi \otimes \Psi}\left(\omega_{0}\right)$, (ii) неравенство (3) имеет место при $\omega=\omega_{n}$ для всех $n \in \mathbb{N}$.

С помощью данной леммы, предложения 7 и теоремы 2 из [7], а также теоремы 1 из [9], получаем бесконечномерный вариант результатов из [9], [6], [8].

ПредЛОжениЕ. Пусть $\Psi$ - произволъный канал. Супераддитивность $\widehat{H}$-функции имеет место в следующих случалх: (i) $\Phi$ - тождественный канал; (ii) $\Phi$ - канал, разрушающий сцепленность (см. [3]); (iii) $\Phi$ - канал, комплементарный (см. [9]) каналу, разрушающему сцепленность; (iv) $\Phi$ - прямая сумма (см. [6]) тождественного канала и канала $\Phi_{0}$ такого, что супераддитивность $\widehat{H}$-функции имеет место для каналов $\Phi_{0} u \Psi$.

Приведенная выше лемма позволяет доказать следующее утверждение.

Теорема 1. Если супераддитивность $\widehat{H}$-функиии имеет место для всех конечномерных квантовых каналов, то это свойство имеет место и для всех бесконечномерных квантовых каналов.

СледСтвие 1. Если супераддитивность ЕоF имеет место для всех состояний конечномерной составной квантовой системы, то это свойство имеет место и для всех состояний бесконечномерной составной квантовой системы.

СледСтвиЕ 2. Если аддитивность минимальной выходной энтропии имеет место для всех конечномерных квантовых каналов, то это свойство имеет место и для всех бесконечномерных квантовых каналов.

Теорема 1 и теорема 3 из [7] позволяют получить бесконечномерный вариант теоремы Шора [2].

Теорема 2. Следующие свойства равносильны: (i) аддитивность х-пропускной способности для всех бесконечноменых каналов с произвольными ограничениями; (ii) аддитивность минимальной выходной энтропии для всех бесконечноменых каналов; (iii) супераддитивность ЕоF для всех состояний произвольной бесконечномерной составной квантовой системы.

Последнее свойство в теореме 2 равносильно супераддитивности $\widehat{H}$-функции для всех бесконечномерных квантовых каналов.

Доказательства всех утверждений данной статьи приведены в [10].

\section{Список литературы}

[1] А.С. Холево, Введение в квантовую теорию информации, МЦНМО, М., 2002. [2] P. W. Shor, Comm. Math. Phys., 246:3 (2004), 453-472; corrections: ibid. 473. [3] A. C. Xoлево, УМН, 61:2 (2006), 113-152. [4] А. Д. Иоффе, В. М. Тихомиров, Теория экстремальных задач, Наука, М., 1974. [5] M. E. Shirokov, arXiv: quant-ph/0411091. [6] A. S. Holevo, M. E. Shirokov, Comm. Math. Phys., 249:2 (2004), 417-430. [7] M. E. Shirokov, Comm. Math. Phys., 262:1 (2006), 137-159. [8] P. W. Shor, J. Math. Phys., 43:9 (2002), 4334-4340. [9] А. С. Холево, Теория вероятн. и ее примен., 51:1 (2006), 133-143. [10] M. E. Shirokov, arXiv: quant-ph/0608090.

М. Е. Широков (М. Е. Shirokov)

Математический институт им. В. А. Стеклова РАН

E-mail: msh@mi.ras.ru
Представлено А. В. Булинским Принято редколлегией 24.10 .2006 